\title{
The Geriatric Nutritional Risk Index Independently Predicts Mortality in Diabetic Foot Ulcers Patients Undergoing Amputations
}

\author{
Yuanyuan Xie, Hailing Zhang, Tingting Ye, Shengjie Ge, Ruyi Zhuo, and Hong Zhu \\ Department of Endocrinology and Metabolism, The First Affiliated Hospital of Wenzhou Medical University, Wenzhou, China \\ Correspondence should be addressed to Hong Zhu; wyyynfm@126.com
}

Received 5 October 2016; Accepted 18 December 2016; Published 9 January 2017

Academic Editor: Patrizio Tatti

Copyright (c) 2017 Yuanyuan Xie et al. This is an open access article distributed under the Creative Commons Attribution License, which permits unrestricted use, distribution, and reproduction in any medium, provided the original work is properly cited.

Objective. Patients with diabetic foot ulcers undergoing amputations have poor prognosis. Malnutrition usually occurs in this population and is associated with increased risk of mortality. The geriatric nutritional risk index (GNRI) is a widely used, simple, and well-established tool to assess nutritional risk. The purpose of this study was to assess the association between GNRI and all-cause mortality in diabetic foot ulcers patients undergoing minor or major amputations. Methods. This was a retrospective cohort study including 271 adult patients. Patients were divided into two groups according to a GNRI cutoff value of 92, and characteristics and mortality were compared between the two groups. Cox proportional hazard analysis was performed to explore the association between GNRI and mortality. Result. GNRI $(p<0.001)$, age $(p<0.001)$, and eGFR $(p=0.002)$ were independent predictors of mortality. Among a subgroup of 230 patients with minor amputation, increased age $(p<0.001)$, coronary artery disease $(p=0.030)$, and increased GNRI $(p<0.001)$ were major risk factors. Conclusion. GNRI on admission might be a novel clinical predictor for the incidence of death in patients with diabetic foot ulcers who were undergoing amputations.

\section{Introduction}

The prevalence of type 2 diabetes has been increasing rapidly throughout the world. In 2015, the International Diabetes Federation estimated that 1 in 11 adults aged between 20 and 79 years had diabetes and that the number of adults worldwide living with diabetes had soared to 425 million. This number is projected to increase to 642 million by 2040 , with a global prevalence of $10.0 \%$ [1]. Diabetic foot ulcers (DFU) are a common complication in diabetic patients, with the lifetime incidence of developing a foot ulcer estimated to be up to $25 \%$ [2]; moreover, DFU are the leading cause of nontraumatic lower extremity amputation (LEA) worldwide [3]. Furthermore, LEA is related to a high risk of death in diabetic subjects $[4,5]$. Diabetic patients undergoing LEA have increased risk of mortality compared with those who have not undergone LEA [6].

A high prevalence of malnutrition, which is frequently unrecognized among patients with chronic or severe diseases, is significantly associated with a longer hospital stay as well as increased morbidity and mortality $[7,8]$. Nutritional deficiencies and poor food intake play a major role in the impaired nutritional status of patients with diabetes-related complications, including renal failure and foot infection [9]. DFU patients, who have a high occurrence of infection and vascular complications, more commonly suffer from malnutrition compared with patients without DFU [10].

A geriatric nutritional risk index (GNRI) has been used as a simple and valuable tool to predict outcomes calculated from only serum albumin and the ratio between actual and ideal body weight [11]. GNRI is a prognostic determinant for clinical outcomes in patients with chronic heart failure [12] and those on hemodialysis [13]. In addition, GNRI has been used as a predictor of amputation in patients with critical limb ischemia $[14,15]$. However, GNRI is rarely adopted to evaluate the nutritional condition of patients suffering from DFU. Moreover, the value of GNRI for predicting mortality in DFU patients who have experienced or are undergoing LEA has not been explored. 
Therefore, the aim of the present study was to determine the predictive relationship between GNRI and prognosis among DFU patients undergoing LEA.

\section{Materials and Methods}

2.1. Study Population. This was a retrospective cohort study including 271 adult patients with diagnoses of type 2 diabetes mellitus and diabetic foot, who had consented to receive minor or major amputation at The First Affiliated Hospital of Wenzhou Medical University between May 2010 and May 2015. Minor amputation was defined as any amputation distal to the ankle joint, and major amputation was defined as amputation above the ankle. The diagnosis of diabetes mellitus was based on World Health Organization criteria or on having been treated with insulin and/or oral hypoglycemic agents. Hypertension was diagnosed if one of the following conditions was present: systolic blood pressure $\geq 140 \mathrm{mmHg}$, diastolic blood pressure $\geq 90 \mathrm{mmHg}$, or the presence of treatment for hypertension. The coronary artery disease was defined by medical history and clinical symptoms of the coronary artery. The cerebral vascular disease was assumed to be present with any event of neurologic deficiency, whether persistent or resolved. Peripheral arterial disease was diagnosed by the presence of stenosis as shown in Doppler ultrasound performed by specialist physicians.

The present study was approved by the ethics committees and was conducted in accordance with Declaration of Helsinki.

2.2. Methods and Calculations. Demographic variables (age and sex), anthropometric parameters (height, weight, and body mass index [BMI]), medical history, history of smoking and alcohol abuse, and comorbidity and laboratory data (e.g., albumin, creatinine) were collected from individual medical records upon admission according to prespecified definitions. Body mass index was calculated as bodyweight divided by height squared $\left(\mathrm{kg} / \mathrm{m}^{2}\right)$. The estimated glomerular filtration rate (eGFR) was calculated according to the Chronic Kidney Disease Epidemiology Collaboration formula, and a physical examination was performed to determine Wagner grade of the foot lesion [16]. Follow-up data were obtained in medical records or by telephone interview. Mortality during follow-up was used as the outcome measure.

GNRI Calculation. GNRI was calculated from individually obtained height in $\mathrm{cm}$, current body weight in $\mathrm{kg}$, ideal body weight, and serum albumin level as follows [11]:

$$
\begin{aligned}
\text { GNRI }=[ & 1489 \times \operatorname{albumin}(\mathrm{g} / \mathrm{L})] \\
& +\left[41.7 \times\left(\frac{\text { weight }}{\text { WLo }}\right)\right],
\end{aligned}
$$

where WLo indicates ideal weight, which was calculated as follows:

$$
\begin{gathered}
\text { men: WLo }=H-100-\left[\frac{(H-150)}{4}\right], \\
\text { women: } H-100-\left[\frac{(H-150)}{2.5}\right],
\end{gathered}
$$

where $H$ indicates height.

From these GNRI values, four grades of nutrition-related risk were defined [11]: high risk $(<82)$, moderate risk (82 to $<92)$, low risk (92-98), and no risk (>98).

2.3. Statistical Analysis. Continuous variables are expressed as mean \pm standard deviation (SD) for normally distributed variables. Variables exhibiting a nonnormal distribution are expressed as median and interquartile range. Categorical variables are expressed as percentages and were compared using the chi-square test. Differences in continuous variables were compared using Student's $t$-test for normally distributed variables and the Whitney $U$ test for nonnormally distributed variables. Survival analysis was estimated using the KaplanMeier method, and differences were compared using the logrank test. The independent association between GNRI and mortality was assessed using the Cox proportional hazard model, which was conducted using stepwise regression. Height, weight, serum albumin, and creatinine were excluded as they were used in the calculation of GNRI, BMI, and eGFR. All analyses were performed using IBM SPSS Statistics version 23. $p<0.05$ was considered statistically significant.

\section{Results}

3.1. Baseline Demographic and Clinical Characteristics. Baseline clinical characteristics and laboratory data for all participants were listed in Table 1 . Mean age was $66.9 \pm 11.1$ years; $59.8 \%$ of participants were men, $93.7 \%$ had peripheral arterial disease, $60.9 \%$ had hypertension, $55.9 \%$ had albuminuria, $14 \%$ had cerebral vascular disease, $9.6 \%$ had coronary artery disease, $19.2 \%$ had lower limb revascularization, $34.7 \%$ were smokers, and $29.2 \%$ were drinkers. Figure 1 shows the distribution of GNRI in the DFU patients undergoing LEA in this study. Mean baseline GNRI was $92.61 \pm 10.00$. Patients were divided into two groups based on the following cutoff value: high GNRI (GNRI $\geq 92, n=138$ ) with low or no nutritional risk and low GNRI (GNRI $<92, n=133)$ with moderate or severe risk.

3.2. Patient Characteristics according to GNRI. As shown in Table 1, the duration of diabetic foot ulcers was longer in the high GNRI group. In addition, BMI, systolic blood pressure, serum hemoglobin, albumin, cholesterol, and high-density lipoprotein (HDL-C) values were significantly higher in high GNRI group. Blood platelet counts, white blood cell counts, low-density lipoprotein (LDL-C) values, and fibrinogen levels were significantly higher in the low GNRI group. The prevalence of smoking was significantly higher in the high GNRI group, and the proportion of major amputation was 
TABLE 1: Baseline patient characteristics.

\begin{tabular}{|c|c|c|c|c|}
\hline & Overall & High GNRI $(\geq 92)$ & Low GNRI $(<92)$ & $p$ value \\
\hline Case $n(\%)$ & 271 & 133 & 138 & \\
\hline Age, years & $66.9 \pm 11.1$ & $67.4 \pm 11.4$ & $66.4 \pm 10.8$ & 0.444 \\
\hline Male $n(\%)$ & $162(59.8)$ & $83(62.4)$ & $79(57.2)$ & 0.387 \\
\hline BMI, kg/m² & $23.0 \pm 3.1$ & $24.4 \pm 2.9$ & $21.7 \pm 2.6$ & $<0.001$ \\
\hline Systolic blood pressure, $\mathrm{mmHg}$ & $243.6 \pm 23.0$ & $147.5 \pm 23.7$ & $139.8 \pm 21.8$ & 0.006 \\
\hline Diastolic blood pressure, $\mathrm{mmHg}$ & $75.0 \pm 11.9$ & $76.0 \pm 11.7$ & $74.0 \pm 12.0$ & 0.155 \\
\hline Duration of diabetes, years & $10.0(4.0-15.0)$ & $10(4.0-15.0)$ & $10.0(5-15.3)$ & 0.197 \\
\hline Duration of diabetic foot ulcers, months & $1.0(0.67-3)$ & $2(0.8-4)$ & $1(0.5-3.0)$ & 0.031 \\
\hline Wagner classification & & & & 0.073 \\
\hline 2 & $14(4.2)$ & $7(5.1)$ & $7(5.3)$ & \\
\hline 3 & $91(33.6)$ & $52(39.1)$ & $39(28.3)$ & \\
\hline 4 & $162(33.6)$ & $74(55.6)$ & $88(63.8)$ & \\
\hline 5 & $4(1.5)$ & $0(0)$ & $4(2.9)$ & \\
\hline \multicolumn{5}{|l|}{ Comorbidity $n(\%)$} \\
\hline Hypertension & $165(60.9)$ & $88(66.2)$ & $77(55.8)$ & 0.080 \\
\hline Cerebral vascular disease & $38(14.0)$ & $17(12.8)$ & $21(15.2)$ & 0.564 \\
\hline Coronary artery disease & $26(9.6)$ & $17(12.8)$ & $9(6.5)$ & 0.080 \\
\hline Peripheral arterial disease & $254(93.7)$ & $127(95.5)$ & $127(92)$ & 0.240 \\
\hline \multicolumn{5}{|l|}{ History $n(\%)$} \\
\hline Smoking (current or ever) & $94(34.7)$ & $55(41.4)$ & $39(28.6)$ & 0.024 \\
\hline Drinking (current or ever) & $79(29.2)$ & $42(31.6)$ & $37(26.8)$ & 0.388 \\
\hline lower limb revascularization & $52(19.2)$ & $30(22.6)$ & $22(15.9)$ & 0.167 \\
\hline \multicolumn{5}{|l|}{ Laboratory date } \\
\hline Hemoglobin, g/L & $108.5 \pm 18.6$ & $113.5 \pm 18.2$ & $103.7 \pm 17.8$ & $<0.001$ \\
\hline White blood cell, $* 10^{9} / \mathrm{L}$ & $8.9(6.9-11.7)$ & $8.23(6.0-10.4)$ & $10.0(7.8-13.01)$ & $<0.001$ \\
\hline Lymphocyte, $* 10^{9} / \mathrm{L}$ & $1.5 \pm 0.7$ & $1.6 \pm 0.7$ & $1.5 \pm 0.6$ & 0.530 \\
\hline Blood platelet $* 10^{9} / \mathrm{L}$ & $276.6 \pm 101.4$ & $248 \pm 85.0$ & $303 \pm 108.6$ & $<0.001$ \\
\hline Albumin, g/L & $33.25 \pm 5.5$ & $36.9 \pm 3.9$ & $29.7 \pm 4.4$ & $<0.001$ \\
\hline Cholesterol, mmol/L & $4.2 \pm 1.2$ & $4.4 \pm 1.1$ & $4.0 \pm 1.2$ & 0.004 \\
\hline Triglycerides, mmol/L & $1.24(0.91-1.24)$ & $1.3(0.9-1.9)$ & $1.2(0.9-1.6)$ & 0.273 \\
\hline HDL-C, mmol/L & $0.9 \pm 0.3$ & $1.0 \pm 0.3$ & $0.8 \pm 0.3$ & $<0.001$ \\
\hline LDL-C, mmol/L & $2.4(1.8-3.0)$ & $2.3(1.8-2.9)$ & $2.5(1.9-3.10)$ & 0.025 \\
\hline Creatinine, $\mu \mathrm{mol} / \mathrm{L}$ & $69(56-92)$ & $71(57-92.5)$ & $67.0(54.0-91.5)$ & 0.353 \\
\hline Blood urea nitrogen, $\mathrm{mmol} / \mathrm{L}$ & $5.8(4.3-7.4)$ & $5.8(4.6-6.9)$ & $5.8(4.2-7.8)$ & 0.941 \\
\hline Uric acid, $\mu \mathrm{mol} / \mathrm{L}$ & $273.9 \pm 103.9$ & $281.6 \pm 89.1$ & $266.44 \pm 116.2$ & 0.230 \\
\hline Fibrinogen, g/L & $6.0 \pm 1.9$ & $5.6 \pm 1.82$ & $6.4 \pm 1.88$ & $<0.001$ \\
\hline Fasting glucose, mmol/L & $8.5(6.1-12.4)$ & $7.9(5.95-11.1)$ & $8.9(6.4-13.05)$ & 0.063 \\
\hline eGFR & $87(62.4-100.3)$ & $85.5(62.0-99.4)$ & $89.8(65.4-102.5)$ & 0.436 \\
\hline Albuminuria & $151(55.9)$ & $71(53.4)$ & $80(58.4)$ & 0.407 \\
\hline Major amputation $n(\%)$ & $41(15.1)$ & $14(10.5)$ & $27(19.6)$ & 0.038 \\
\hline
\end{tabular}

Data are presented as either means \pm standard deviation or median (interquartile range) for continuous variables or number (\%) for categorical variables. GNRI, geriatric nutritional risk index; BMI, body mass index; eGFR, estimated glomerular filtration rate; HDL-C, high-density lipoprotein cholesterol; LDLC, low-density lipoprotein cholesterol.

significantly higher in the low GNRI group. There were no statistically significant between-group differences in eGFR; age; sex; comorbidity; history of lower limb revascularization; drinking; albuminuria; Wagner classification; lymphocyte, triglycerides, and creatinine; blood urea nitrogen, uric acid, fasting glucose levels, or systolic blood pressure.
3.3. Causes of Death. Of the 271 subjects who completed the follow-up, a total of $72(26.6 \%)$ subjects died. The causes of death included the following: 22 (30.1\%) foot-related deaths, $13(18.0 \%)$ deaths due to cardiac disease, 10 (13.9\%) deaths due to cerebrovascular disease, $9(12.5 \%)$ deaths due to renal failure, 7 (9.7\%) deaths due to infections, 2 (2.8\%) deaths 
TABLE 2: Independent predictor of all-cause mortality in DFU with amputations.

\begin{tabular}{|c|c|c|c|c|}
\hline & \multicolumn{2}{|c|}{ Univariate analysis } & \multicolumn{2}{|c|}{ Stepwise multivariate analysis } \\
\hline & $\operatorname{HR}(95 \% \mathrm{CI})$ & $p$ value & $\operatorname{HR}(95 \% \mathrm{CI})$ & $p$ value \\
\hline Age (years) & $1,044(1.021-1.068)$ & $<0.001$ & $1.046(1.022-1.072)$ & $<0.001$ \\
\hline BMI & $0.918(0.850-0.991)$ & 0.029 & & \\
\hline Hypertension & $1.867(1.121-3.109)$ & 0.016 & & \\
\hline Hemoglobin, g/L & $0.985(0.972-0.998)$ & 0.028 & & \\
\hline Albumin, $g / L$ & $0.945(0.906-0.985)$ & 0.008 & & \\
\hline Creatinine, $\mu \mathrm{mol} / \mathrm{L}$ & $1.002(1.001-1.003)$ & 0.001 & & \\
\hline eGFR & $0.985(0.977-0.992)$ & $<0.001$ & $0.987(0.979-0.995)$ & 0.002 \\
\hline GNRI & $0.960(0.938-0.983)$ & 0.001 & $0.945(0.921-0.971)$ & $<0.001$ \\
\hline
\end{tabular}

HR, hazard ratio; CI, confidence interval. Other abbreviations are as in Table 1.

In the multivariate model, the following variables were added as independent variables: age, sex, history of hypertension, albuminuria hemoglobin level, estimated glomerular filtration rate, the presence of minor or major amputation, Wagner classification, and GNRI.

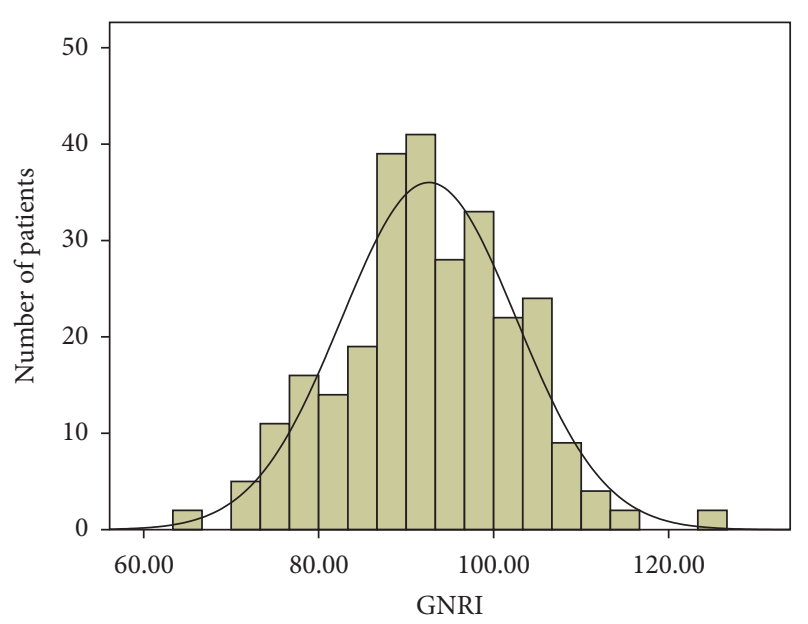

FIGURE 1: Distribution of geriatric nutritional risk index (GNRI).

due to cancer-related death, $2(2.8 \%)$ deaths due to acute complication of diabetes, and $7(9.7 \%)$ deaths with other causes.

3.4. Predictors of Mortality after Amputation. Kaplan-Meier survival analysis for all-cause mortality is shown in Figure 2. The overall survival rate was significantly lower in the low GNRI group (log-rank $p<0.001)$. A significant increase in the incidence of death was observed in patients with increased eGFR (log-rank $p<0.001)$. Mortality rates at $1,3,5$, and 6 years after amputation were 11\%, 27\%, 41\%, and 47\%, respectively. Mean survival times (months) in the low GNRI group were $45.797 \pm 2.568$ (95\% CI 40.763-50.831) and in the high GNRI group were $60.084 \pm 2.151$ (95\% CI $55.868-$ 64.301).

As shown in Table 2, univariate analysis revealed that the following variables were significantly associated with mortality: age (hazard ratio 1.044, 95\% CI 1.021-1.068, and $p<0.001$ ), BMI (hazard ratio 0.918, 95\% CI 0.850-0.991, and $p=0.029$ ), hypertension (hazard ratio $1.867,95 \%$ CI $1.121-$ 3.109 , and $p=0.016$ ), hemoglobin (hazard ratio $0.985,95 \%$ CI $0.972-0.998$, and $p=0.028$ ), albumin (hazard ratio 0.945 ,
95\% CI 0.906-0.985, and $p=0.008$ ), creatinine (hazard ratio $1.002,95 \%$ CI 1.001-1.003, and $p=0.001$ ), eGFR (hazard ratio $0.985,95 \%$ CI $0.977-0.992$, and $p<0.001)$, and GNRI (hazard ratio $0.960,95 \%$ CI $0.938-0.983$, and $p=0.001$ ). In the multivariate Cox proportional hazard model analysis, GNRI as a continuous variable (hazard ratio $0.945,95 \% \mathrm{CI}$ $0.921-0.971$, and $p<0.001$ ) and age (hazard ratio $1.046,95 \%$ CI 1.022-1.071, $p<0.001$ ) and eGFR (hazard ratio $0.987,95 \%$ CI $0.979-0.995, p=0.002$ ) were independent predictors of mortality.

3.5. Predictors of Mortality after Minor Amputation. As shown in Table 3, univariate analysis revealed significant association with mortality for the following variables: age (hazard ratio 1.053, 95\% CI 1.027-1.079, and $p<0.001$ ), cerebral vascular disease (hazard ratio $1.979,95 \%$ CI $1.070-$ 3.662 , and $p=0.016$ ), coronary artery disease (hazard ratio 2.286, 95\% CI 1.119-4.671, and $p=0.023$ ), creatinine (hazard ratio $1.002,95 \%$ CI 1.001-1.004, and $p=0.008$ ), eGFR (hazard ratio $0.986,95 \%$ CI $0.977-0.994$, and $p=0.001)$, and GNRI (hazard ratio $0.960,95 \%$ CI $0.935-0.987$, and $p=0.001$ ). In the multivariate Cox proportional hazard model analysis, GNRI as a continuous variable (hazard ratio $0.936,95 \% \mathrm{CI}$ $0.908-0.965$, and $p<0.001$ ), age (hazard ratio $1.061,95 \%$ CI 1.033-1.089, and $p<0.001)$, and coronary artery disease (hazard ratio $2.291,95 \%$ CI $1.086-4.836$, and $p=0.030$ ) were independent predictors of mortality.

\section{Discussion}

In the present study, we investigated the validity of GNRI for predicting all-cause death among DFU patients who underwent LEA. To the best of our knowledge, this is the first study demonstrating that lower GNRI before surgery was significantly associated with higher mortality among these patients. As expected, DFU patients undergoing LEA in the current study had high mortality (mean survival 53.8 months). This agrees with data from previous reports of high mortality rates in diabetic patients with amputations $[4,17]$. From the current and prior results, we propose using GNRI for nutritional evaluation to predict mortality and provide a basis for appropriate and timely nutritional treatment in DFU patients. 


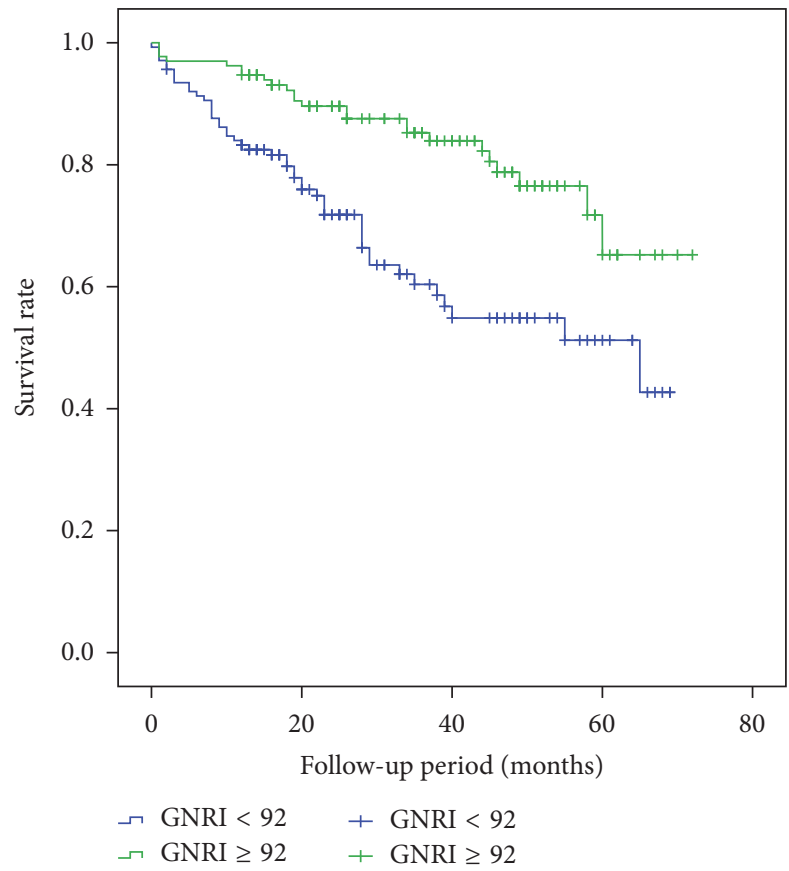

(a)

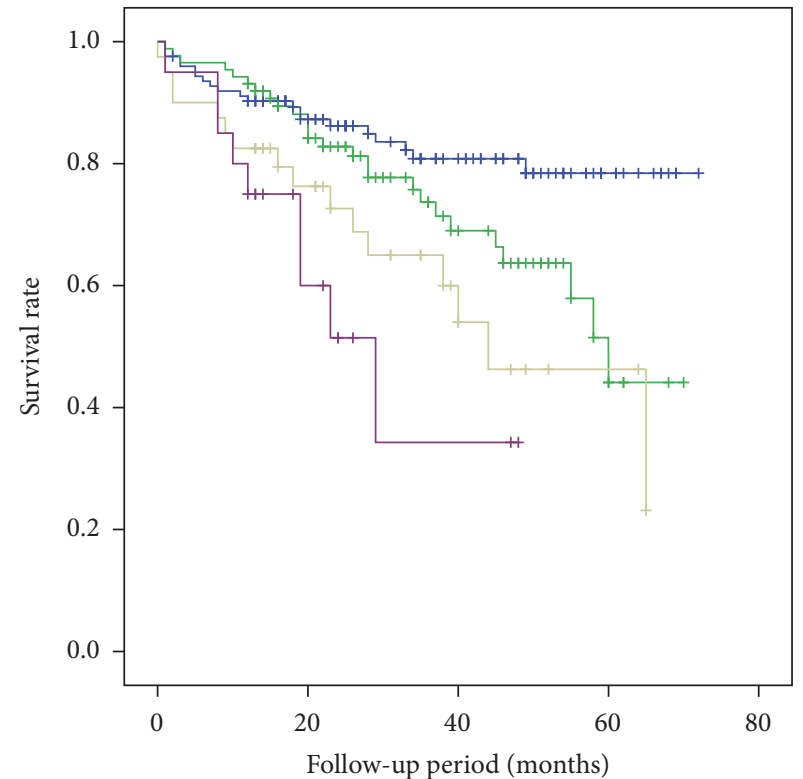

$$
\begin{array}{ll}
\neg \mathrm{eGFR} \geq 90 & +\mathrm{eGFR} \geq 90 \\
\neg 60 \leq \mathrm{eGFR}<90 & +60 \leq \mathrm{eGFR}<90 \\
\neg 30 \leq \mathrm{eGFR}<60 & +30 \leq \mathrm{eGFR}<60 \\
\neg \mathrm{eGFR}<30 & +\mathrm{eGFR}<30
\end{array}
$$

(b)

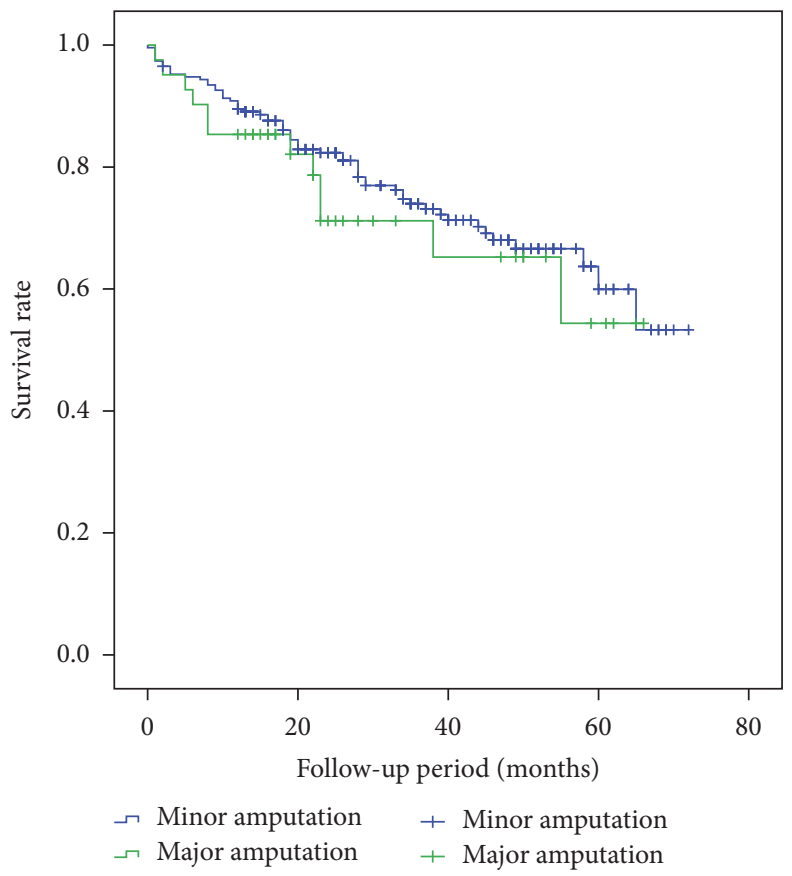

(c)

FIGURE 2: Kaplan-Meier survival curves of the proportion of patients with mortality, according to (a) GNRI categories (low GNRI, <92; high GNRI, $\geq 92$ ), (b) eGFR stratification (eGFR, $\geq 90 ; 60 \leq$ eGFR $<90 ; 30 \leq$ eGFR $<60$; eGFR < 30), or (c) amputation categories (minor amputation; major amputation). The differences between groups in (a) and (b) were significant (log-rank test, all $p<0.001$ ). There was no significant difference in (c) between groups (log-rank test, $p=0.496$ ). 
TABLE 3: Independent predictor of all-cause mortality in DFU with minor amputations.

\begin{tabular}{|c|c|c|c|c|}
\hline & \multicolumn{2}{|c|}{ Univariate analysis } & \multicolumn{2}{|c|}{ Stepwise multivariate analysis } \\
\hline & $\operatorname{HR}(95 \% \mathrm{CI})$ & $p$ value & $\operatorname{HR}(95 \% \mathrm{CI})$ & $p$ value \\
\hline Age (years) & $1,053(1.027-1.079)$ & $<0.001$ & $1.061(1.033-1.089)$ & $<0.001$ \\
\hline BMI & $0.918(0.850-0.991)$ & 0.029 & & \\
\hline Cerebral vascular disease & $1.979(1.070-3.662)$ & 0.016 & & \\
\hline Coronary artery disease & $2.286(1.119-4.671)$ & 0.023 & $2.291(1.086-4.836)$ & 0.030 \\
\hline Creatinine, $\mu \mathrm{mol} / \mathrm{L}$ & $1.002(1.001-1.004)$ & 0.008 & & \\
\hline eGFR & $0.986(0.977-0.994)$ & 0.001 & & \\
\hline GNRI & $0.960(0.935-0.987)$ & 0.003 & $0.936(0.908-0.965)$ & $<0.001$ \\
\hline
\end{tabular}

HR, hazard ratio; CI, confidence interval. Other abbreviations are as in Table 1.

In the multivariate model, the following variables were added as independent variables: age, sex, hemoglobin, history of hypertension, cerebral vascular disease, coronary artery disease, albuminuria hemoglobin level, estimated glomerular filtration rate, Wagner classification, and GNRI.

Previous studies have demonstrated that older age and a higher level of amputation have a worse prognosis than do minor amputees [18-20]. However, the study from Taiwan found that major amputation is not an associated risk factor for mortality after adjusting for sex and age [21]. Another study demonstrated that there is no difference in prognosis between major and minor amputations at two years if the surgical wounds could heal [22]. In our study, wounds healed after amputations among most patients, which might explain that the level of amputation was not associated with mortality. Furthermore, whether the surgical procedure of minor LEA was too conservative in some severe cases who should have had a higher level of amputation was not known. Our findings again stress the point that the coronary artery disease [22] and renal function [18] are associated with mortality in diabetic patients after lower extremity amputations.

Bouillanne et al. were the first to assess that GNRI was predictive of mortality in hospitalized elderly patients [11]. Subsequently, the predictive value of GNRI for prognosis has also been demonstrated for older age, dialysis patients, and cardiovascular patients [10-13]. The Mini-Nutritional Assessment (MNA) is a well-validated nutritional screening tool with high sensitivity and specificity and is widely used in clinical practice to measure nutritional status [23]. GNRI has a poorer tendency to classify patients as being at risk or malnourished than MNA but appears to better predict outcomes [24-27]. According to these studies, GNRI is not an index of malnutrition but is useful as a nutrition-related risk index based on GNRI cutoff values. Therefore, it is reasonable to consider that adoption of GNRI is appropriate for predicting risk of mortality and morbidity.

Malnutrition is common in patients with limb-threatening DFU and has a significant influence on prognosis [10], including delayed wound healing [28], longer hospital stay, and increased mortality [7, 8, 29]. Moreover, as nutritional status is related to immunity, patients with poor nutritional status more commonly suffer from infection [30]. The weakened immune system in these malnourished patients may help to explain the high rate of infection-related complications and mortality.

Foot infections are a consequence of foot ulceration and have the potential to expand and deepen, which can result in loss of limb [31]. Moreover, the inflammatory response to foot infection leads to high metabolism, which requires more nutrients and is associated with protein deficiency during the wound healing process [28]. Consistent with this, serum albumin level is lower in DFU patients who required amputation [32]. Mean serum albumin in the current study was $33.25 \mathrm{~g} / \mathrm{L}$, which is lower than normal serum concentration. In general, patients with more severe disease have a lower serum albumin concentration [33].

Serum albumin is considered as a clinical monitoring tool for nutritional assessment, and hypoalbuminemia is strongly correlated with complications and mortality in the elderly [34-36]. Therefore, hypoalbuminemia is considered as a predictive risk of mortality. With advancing age, serum albumin level gradually decreases [37], suggesting an impaired ability to adapt to disease-related metabolic stress in elderly persons [25]. The present results confirm that poor clinical outcome is associated with hypoalbuminemia in patients who may not have been able to cope with the metabolic stress of foot infection. Furthermore, albumin is an important factor in the GNRI formula and thus may potentially explain the relationship between GNRI and mortality among DFU patients undergoing LEA.

Clinically, pain from ulcers and surgery may prevent physical activity, resulting in disuse atrophy of skeletal muscle. In addition, lower dietary intake and increased nutrient needs, which paradoxically exist in association with diseases, lead to muscle catabolism with subsequent negative effects on muscle strength [38]. Furthermore, higher levels of inflammation markers, which are commonly found in diabetes [39], are associated with decreased muscle strength and function [40]. Therefore, diabetes accelerates the loss of muscle strength and quality [39]. In addition, there is also a clear inverse relationship between muscle strength and mortality [41]. Furthermore, GNRI is predictive of muscle dysfunction [42, 43]. Accordingly, the relationship between low GNRI and high mortality may involve impaired muscle strength.

A number of therapies are available to reduce ulcer risk, heal wounds, and prevent amputations [31, 44, 45]. Unfortunately, LEA is often performed as the ultimate endpoint of diabetic foot disease, and current treatments for these patients are not as effective as they should be. On the other hand, in patients after surgery or injury, the hypermetabolic response persists for a long time, leading to large losses of 
protein from skeletal muscle [46]. Based on protein intake from the typical three daily meals, patients may not consume sufficient nitrogen to meet their hypermetabolic need.

We suggest the necessity of appropriate and timely nutritional support to improve clinical outcomes, especially reduced mortality. There is evidence that such intervention may be successful. For example, in a group of patients at risk of malnutrition, losses in muscle strength and weight were prevented by nutritional supplementation [47].

Moreover, dynamic changes in serum albumin have been observed from admission to discharge [35]. Throughout hospitalization, hypoalbuminemia develops or is maintained if present on admission. Improvement of serum albumin from low to normal levels may help decrease mortality. Because nutritional status may be improved with appropriate interventions, this may help maintain muscular functional capacity, improve serum albumin level, and recover immune function, although additional studies are needed to verify this. We conclude that GNRI should be used to evaluate the appropriate nutritional intervention.

There are several limitations to the present study. First, it was a single-center study with a relatively small sample size; thus the results may not be representative for patients with diabetes-related amputations in general. Second, the GNRI values were not measured with a disease-specific scale, such as the diabetic foot disease scale, which would provide a more adequate measurement. Third, it remains unclear how high malnutrition risk reflects the prevalence of malnutrition. As mentioned above, GNRI is a nutrition-related risk index. Unfortunately, detailed nutritional and other information, such as dietary intake, weight loss, and muscle strength or mass, was not obtained in the current study. Furthermore, there is currently no gold standard for diagnosing malnutrition. Fourth, we only analyzed the predictive value of GNRI before surgery and did not analyze the impact of dynamic changes in GNRI on mortality. The present findings need to be confirmed by further studies, and future prospective studies with a larger sample size and a multicenter design should evaluate if disease-specific GNRI is associated with clinical outcomes.

In conclusion, GNRI on admission is an effective predictive marker for mortality in DFU patients undergoing LEA.

\section{Competing Interests}

The authors declare that they have no conflict of interests.

\section{Authors' Contributions}

Hailing Zhang contributed to statistical analysis. Tingting Ye, Shengjie Ge, and Ruyi Zhuo contributed to study design and data collection. All authors read and approved the final paper. All authors participated in the overall design of the study, interpretation of results, and drafting of the manuscript.

\section{Acknowledgments}

The authors thank the staff at the Department of Endocrinology and Metabolism, the First Affiliated Hospital of Wenzhou
Medical University, and all the patients who participated in the study.

\section{References}

[1] IDF, IDF Diabetes Atlas, International Diabetes Federation, Brussels, Belgium, 7th edition, 2015.

[2] N. Singh, D. G. Armstrong, and B. A. Lipsky, "Preventing foot ulcers in patients with diabetes," Journal of the American Medical Association, vol. 293, no. 2, pp. 217-228, 2005.

[3] A. J. Boulton, L. Vileikyte, G. Ragnarson-Tennvall, and J. Apelqvist, "The global burden of diabetic foot disease," The Lancet, vol. 366, no. 9498, pp. 1719-1724, 2005.

[4] N. Tentolouris, S. Al-Sabbagh, M. G. Walker, A. J. M. Boulton, and E. B. Jude, "Mortality in diabetic and nondiabetic patients after amputations performed from 1990 to 1995: a 5-year followup study," Diabetes Care, vol. 27, no. 7, pp. 1598-1604, 2004.

[5] D. Martins-Mendes, M. Monteiro-Soares, E. J. Boyko et al., "The independent contribution of diabetic foot ulcer on lower extremity amputation and mortality risk," Journal of Diabetes and its Complications, vol. 28, no. 5, pp. 632-638, 2014.

[6] O. Hoffstad, N. Mitra, J. Walsh, and D. J. Margolis, "Diabetes, lower-extremity amputation, and death," Diabetes Care, vol. 38, no. 10, pp. 1852-1857, 2015.

[7] K. Norman, C. Pichard, H. Lochs, and M. Pirlich, "Prognostic impact of disease-related malnutrition," Clinical Nutrition, vol. 27, no. 1, pp. 5-15, 2008.

[8] M. I. T. D. Correia and D. L. Waitzberg, "The impact of malnutrition on morbidity, mortality, length of hospital stay and costs evaluated through a multivariate model analysis," Clinical Nutrition, vol. 22, no. 3, pp. 235-239, 2003.

[9] M. Elia, A. Ceriello, H. Laube, A. J. Sinclair, M. Engfer, and R. J. Stratton, "Enteral nutritional support and use of diabetes-specific formulas for patients with diabetes: a systematic review and meta-analysis," Diabetes Care, vol. 28, no. 9, pp. 2267-2279, 2005.

[10] S.-S. Zhang, Z.-Y. Tang, P. Fang, H.-J. Qian, L. Xu, and G. Ning, "Nutritional status deteriorates as the severity of diabetic foot ulcers increases and independently associates with prognosis," Experimental and Therapeutic Medicine, vol. 5, no. 1, pp. 215222, 2013.

[11] O. Bouillanne, G. Morineau, C. Dupant et al., "Geriatric nutritional risk index: a new index for evaluating at-risk elderly medical patients," American Journal of Clinical Nutrition, vol. 82, no. 4, pp. 777-783, 2005.

[12] Y. Kinugasa, M. Kato, S. Sugihara et al., "Geriatric nutritional risk index predicts functional dependency and mortality in patients with heart failure with preserved ejection fraction," Circulation Journal, vol. 77, no. 3, pp. 705-711, 2013.

[13] I. Kobayashi, E. Ishimura, Y. Kato et al., "Geriatric Nutritional Risk Index, a simplified nutritional screening index, is a significant predictor of mortality in chronic dialysis patients," Nephrology Dialysis Transplantation, vol. 25, no. 10, pp. 3361-3365, 2010.

[14] T. Shiraki, O. Iida, M. Takahara et al., “The geriatric nutritional risk index is independently associated with prognosis in patients with critical limb ischemia following endovascular therapy," European Journal of Vascular and Endovascular Surgery, vol. 52, no. 2, pp. 218-224, 2016. 
[15] H. Luo, H. Yang, B. Huang, D. Yuan, J. Zhu, and J. Zhao, "Geriatric nutritional risk index (GNRI) independently predicts amputation inchronic criticallimb ischemia (CLI)," PLoS ONE, vol. 11, no. 3, Article ID e0152111, 2016.

[16] A. Karthikesalingam, P. J. E. Holt, P. Moxey, K. G. Jones, M. M. Thompson, and R. J. Hinchliffe, "A systematic review of scoring systems for diabetic foot ulcers," Diabetic Medicine, vol. 27, no. 5, pp. 544-549, 2010.

[17] S. Morbach, U. Gröblinghoff, H. Schulze et al., "All-cause mortality after diabetes-related amputation in barbados: a prospective case-control study: response to Hambleton et al," Diabetes Care, vol. 32, no. 8, article e100, pp. 306-307, 2009.

[18] D. C. Jupiter, J. C. Thorud, C. J. Buckley, and N. Shibuya, “The impact of foot ulceration and amputation on mortality in diabetic patients. I: from ulceration to death, a systematic review," International Wound Journal, vol. 23, no. 1, pp. 7-11, 2015.

[19] A. Papazafiropoulou, N. Tentolouris, R.-P. Soldatos et al., "Mortality in diabetic and nondiabetic patients after amputations performed from 1996 to 2005 in a tertiary hospital population: a 3-year follow-up study," Journal of Diabetes and Its Complications, vol. 23, no. 1, pp. 7-11, 2009.

[20] Y. Izumi, K. Satterfield, S. Lee, L. B. Harkless, and L. A. Lavery, "Mortality of first-time amputees in diabetics: a 10-year observation," Diabetes Research and Clinical Practice, vol. 83, no. 1, pp. 126-131, 2009.

[21] C.-H. Tseng, C.-K. Chong, C.-P. Tseng, J.-C. Cheng, M.-K. Wong, and T.-Y. Tai, "Mortality, causes of death and associated risk factors in a cohort of diabetic patients after lower-extremity amputation: a 6.5-year follow-up study in Taiwan," Atherosclerosis, vol. 197, no. 1, pp. 111-117, 2008.

[22] K. Matsuzaki, R. Hayashi, K. Okabe, N. Aramaki-Hattori, and K. Kishi, "Prognosis of critical limb ischemia: major vs. minor amputation comparison," Wound Repair and Regeneration, vol. 23, no. 5, pp. 759-764, 2015.

[23] B. Vellas, H. Villars, G. Abellan et al., "Overview of the MNA ${ }^{\circledR}$ its history and challenges," Journal of Nutrition, Health and Aging, vol. 10, no. 6, pp. 456-463, 2006.

[24] E. Cereda, C. Pusani, D. Limonta, and A. Vanotti, "The ability of the Geriatric Nutritional Risk Index to assess the nutritional status and predict the outcome of home-care resident elderly: a comparison with the Mini Nutritional Assessment," British Journal of Nutrition, vol. 102, no. 4, pp. 563-570, 2009.

[25] W. M. Abd-El-Gawad, R. M. Abou-Hashem, M. O. El Maraghy, and G. E. Amin, "The validity of geriatric nutrition risk index: simple tool for prediction of nutritional-related complication of hospitalized elderly patients. Comparison with Mini Nutritional Assessment," Clinical Nutrition, vol. 33, no. 6, pp. 11081116, 2014.

[26] P. Durán Alert, R. Milà Villarroel, F. Formiga, N. Virgili Casas, and C. Vilarasau Farré, "Assessing risk screening methods of malnutrition in geriatric patients: Mini Nutritional Assessment (MNA) versus Geriatric Nutritional Risk Index (GNRI)," Nutricion Hospitalaria, vol. 27, no. 2, pp. 590-598, 2012.

[27] S. Gärtner, M. Kraft, J. Krüger et al., "Geriatric nutritional risk index correlates with length of hospital stay and inflammatory markers in older inpatients," Clinical Nutrition, 2016.

[28] C. Legendre, C. Debure, S. Meaume, C. Lok, J. L. Golmard, and P. Senet, "Impact of protein deficiency on venous ulcer healing," Journal of Vascular Surgery, vol. 48, no. 3, pp. 688-693, 2008.
[29] E. Cereda, C. Pedrolli, A. Zagami et al., "Nutritional risk, functional status and mortality in newly institutionalised elderly," British Journal of Nutrition, vol. 110, no. 10, pp. 1903-1909, 2013.

[30] G. T. Keusch, "The history of nutrition: malnutrition, infection and immunity," Journal of Nutrition, vol. 133, no. 1, pp. 336S340S, 2003.

[31] D. G. Armstrong and B. A. Lipsky, "Diabetic foot infections: stepwise medical and surgical management," International Wound Journal, vol. 1, no. 2, pp. 123-132, 2004.

[32] D. K. Wukich, K. B. Hobizal, and M. M. Brooks, "Severity of diabetic foot infection and rate of limb salvage," Foot and Ankle International, vol. 34, no. 3, pp. 351-358, 2013.

[33] P. E. Ballmer, "Causes and mechanisms of hypoalbuminaemia," Clinical Nutrition, vol. 20, no. 3, pp. 271-273, 2001.

[34] S. Cabrerizo, D. Cuadras, F. Gomez-Busto, I. Artaza-Artabe, F. Marín-Ciancas, and V. Malafarina, "Serum albumin and health in older people: review and meta analysis," Maturitas, vol. 81, no. 1, pp. 17-27, 2015.

[35] D. Barchel, D. Almoznino-Sarafian, M. Shteinshnaider, I. Tzur, N. Cohen, and O. Gorelik, "Clinical characteristics and prognostic significance of serum albumin changes in an internal medicine ward," European Journal of Internal Medicine, vol. 24, no. 8, pp. 772-778, 2013.

[36] G. Franch-Arcas, "The meaning of hypoalbuminaemia in clinical practice," Clinical Nutrition, vol. 20, no. 3, pp. 265-269, 2001.

[37] G. Sergi, A. Coin, G. Enzi et al., "Role of visceral proteins in detecting malnutrition in the elderly," European Journal of Clinical Nutrition, vol. 60, pp. 203-209, 2006.

[38] J. M. Argilés, N. Campos, J. M. Lopez-Pedrosa, R. Rueda, and L. Rodriguez-Mañas, "Skeletal muscle regulates metabolism via interorgan crosstalk: roles in health and disease," Journal of the American Medical Directors Association, vol. 17, no. 9, pp. 789796, 2016.

[39] W. P. Seok, B. H. Goodpaster, E. S. Strotmeyer et al., "Accelerated loss of skeletal muscle strength in older adults with type 2 diabetes: the health, aging, and body composition study, Diabetes Care, vol. 30, no. 6, pp. 1507-1512, 2007.

[40] M. Visser, M. Pahor, D. R. Taaffe et al., "Relationship of interleukin- 6 and tumor necrosis factor- $\alpha$ with muscle mass and muscle strength in elderly men and women: the health $\mathrm{ABC}$ study," Journals of Gerontology A, vol. 57, no. 5, pp. M326-M332, 2002.

[41] A. B. Newman, V. Kupelian, M. Visser et al., "Strength, but not muscle mass, is associated with mortality in the health, aging and body composition study cohort," The Journals of Gerontology Series A: Biological Sciences and Medical Sciences, vol. 61, no. 1, pp. 72-77, 2006.

[42] E. Cereda and A. Vanotti, “The new Geriatric Nutritional Risk Index is a good predictor of muscle dysfunction in institutionalized older patients," Clinical Nutrition, vol. 26, no. 1, pp. 78-83, 2007.

[43] E. Cereda and A. Vanotti, "Short dietary assessment improves muscle dysfunction identification by Geriatric Nutritional Risk Index in uncomplicated institutionalised patients over 70 years old," Clinical Nutrition, vol. 27, no. 1, pp. 126-132, 2008.

[44] N. C. Schaper, J. J. Van Netten, J. Apelqvist, B. A. Lipsky, and K. Bakker, "Prevention and management of foot problems in diabetes: a summary guidance for daily practice 2015, based on the IWGDF guidance documents," Diabetes/Metabolism Research and Reviews, vol. 32, pp. 7-15, 2016. 
[45] M. P. Khanolkar, S. C. Bain, and J. W. Stephens, "The diabetic foot," QJM, vol. 101, no. 9, pp. 685-695, 2008.

[46] D. N. Monk, L. D. Plank, G. Franch-Arcas, P. J. Finn, S. J. Streat, and G. L. Hill, "Sequential changes in the metabolic response in critically injured patients during the first 25 days after blunt trauma," Annals of Surgery, vol. 223, no. 4, pp. 395-405, 1996.

[47] M. Persson, Å. Hytter-Landahl, K. Brismar, and T. Cederholm, "Nutritional supplementation and dietary advice in geriatric patients at risk of malnutrition," Clinical Nutrition, vol. 26, no. 2, pp. 216-224, 2007. 


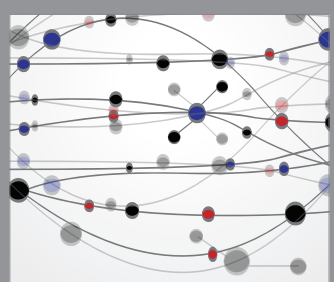

The Scientific World Journal
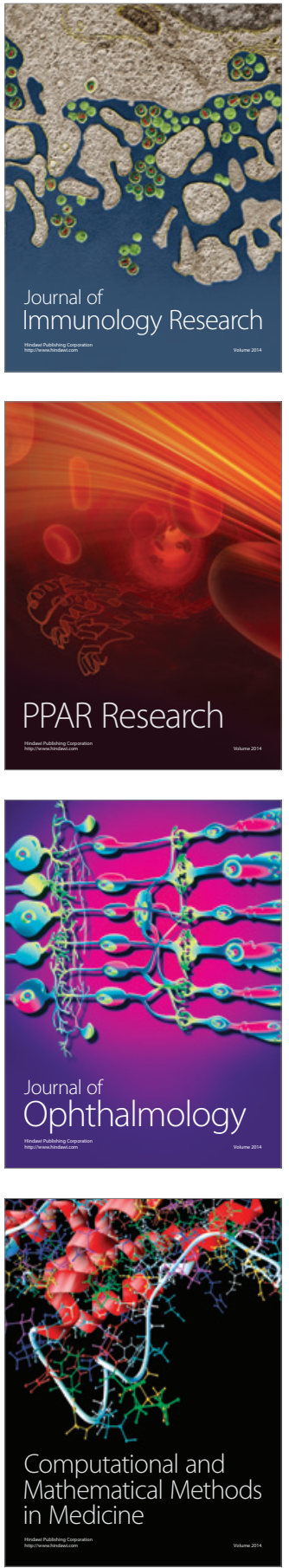

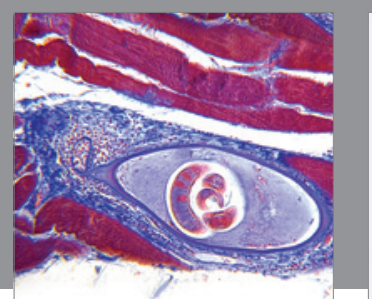

Gastroenterology Research and Practice
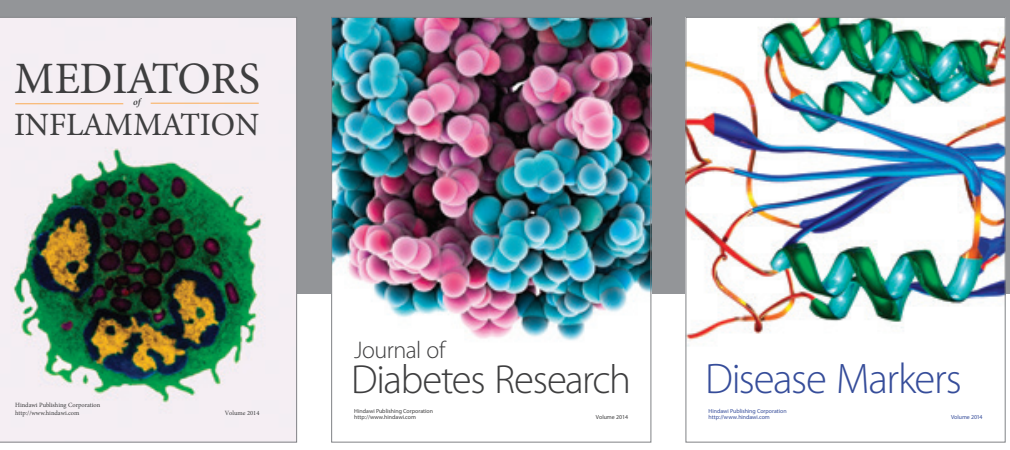

Disease Markers

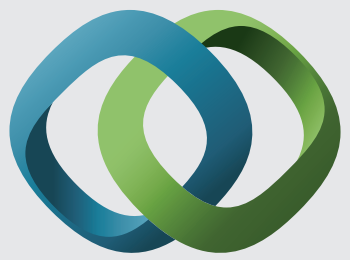

\section{Hindawi}

Submit your manuscripts at

https://www.hindawi.com
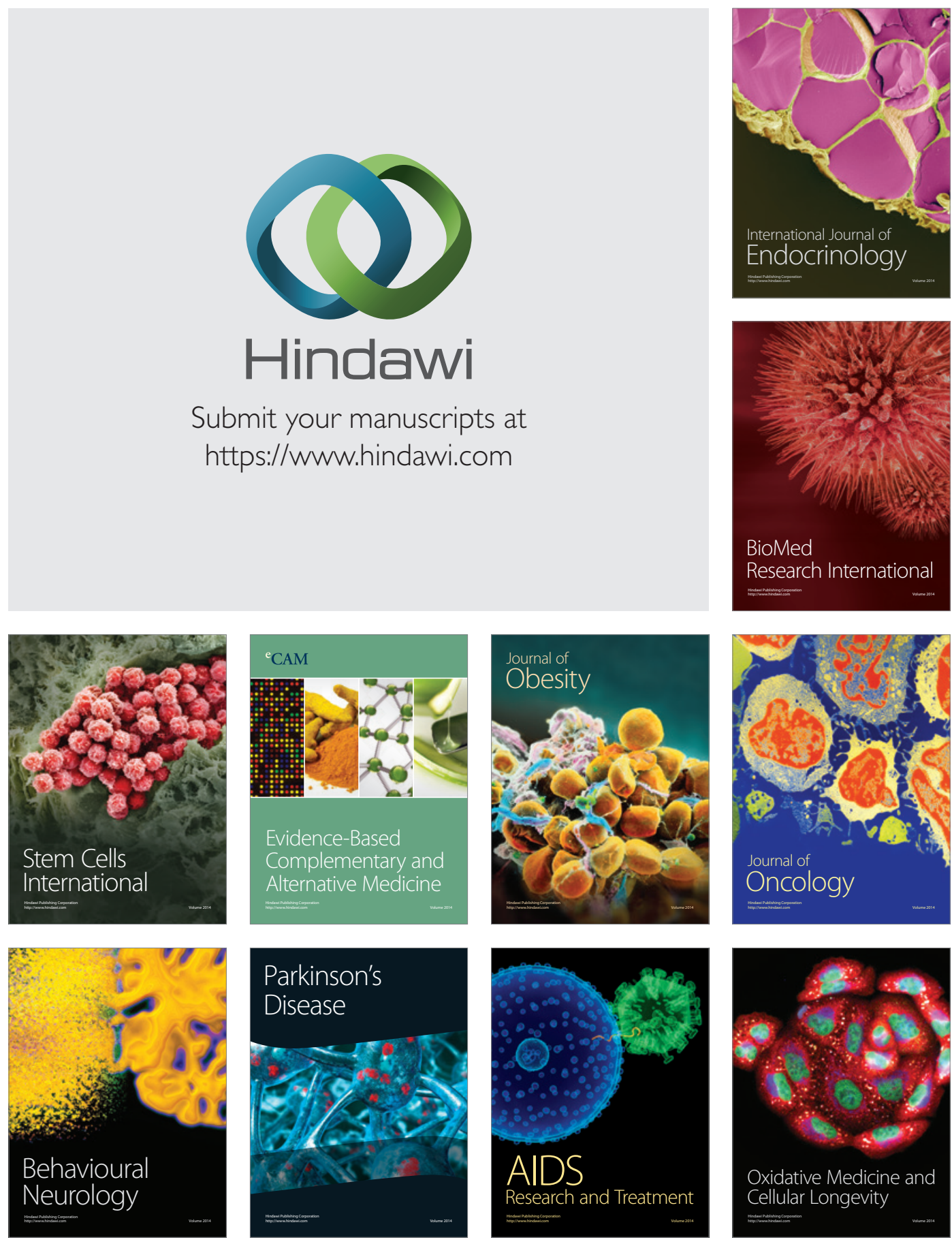